\title{
Frontières
}

\section{André Compte-Sponville, Présentations de la philosophie, Paris, Albin Michel, 2000, 219 pages}

\section{Jean-Jacques Lavoie}

Volume 13, numéro 1, automne 2000

URI : https://id.erudit.org/iderudit/1074263ar

DOI : https://doi.org/10.7202/1074263ar

Aller au sommaire du numéro

Éditeur(s)

Université du Québec à Montréal

ISSN

1180-3479 (imprimé)

1916-0976 (numérique)

Découvrir la revue

Citer ce compte rendu

Lavoie, J.-J. (2000). Compte rendu de [André Compte-Sponville, Présentations de la philosophie, Paris, Albin Michel, 2000, 219 pages]. Frontières, 13(1), 86-87.

https://doi.org/10.7202/1074263ar

Ce document est protégé par la loi sur le droit d'auteur. L'utilisation des services d’Érudit (y compris la reproduction) est assujettie à sa politique d'utilisation que vous pouvez consulter en ligne.

https://apropos.erudit.org/fr/usagers/politique-dutilisation/
Cet article est diffusé et préservé par Érudit.

Érudit est un consortium interuniversitaire sans but lucratif composé de l’Université de Montréal, l’Université Laval et l’Université du Québec à Montréal. Il a pour mission la promotion et la valorisation de la recherche. https://www.erudit.org/fr/ 
ginaire où il peut communiquer avec les autres et avec lui-même et où il peut penser librement à la mort sur un mode magique. Le recours à la mort, comme visa pour le pays de la fin heureuse, est d'abord une stratégie en faveur de la vie. Puis, vient le moment où le jeune s'aperçoit que la jolie mort se transforme en vraie mort. Perplexe et décontenancé, il entre dans la phase de lutte où, avec l'énergie du désespoir, il cherche à venir à bout de cette nécessité indésirable et à tuer la cause de ses tourments, à reconquérir la vie et à restaurer les moments heureux du passé. Cependant, sa mémoire est courte et le temps l'épuise. Alors, il se détourne de la réalité trop dure pour lui et il abandonne le combat. Il aborde la phase de renoncement où, avec tristesse et désarroi, il se soumet à son destin mortel. II n'est pas encore prêt à se tuer, mais il désire mourir ou être tué, tuer l'autre en soi, disparaître ou se désintégrer. Il aspire à la mort comme à " une sorte d'euthanasie » (p. 54), comme délivrance ou remède contre une insupportable souffrance. Puis approche l'heure où il décide de tuer le temps et de programmer sa propre fin. Il va jusqu'à préparer le scénario, repérer le lieu ou l'arme du crime. Le passage à l'acte devient une simple formalité. Cet état correspond à la phase du ressentiment où la toute-puissance est à son apogée et où, convaincu de l'inutilité de l'existence, il croit pouvoir maîtriser la mort pour en faire une œuvre d'art ou même pour entraîner autrui à le suivre. Enfin, dans la phase de l'œil du cyclone, l'adolescent vit dans un état de conscience altérée ou de transe. Il se sent comme déjà mort et il dispose d'un temps indéterminé pour se tuer. Cette indétermination peut être une porte de salut. La séquence des phases n'est donc pas irréversible. D'ailleurs, à chacune des phases, l'adolescent peut être aidé à trouver des repères extérieurs ou à puiser dans ses ressources afin de briser le linéaire de la mort.

\section{Le décryptage des signaux}

"En prêtant attention à ce que dit l'adolescent de la réalité, nous pouvons le situer sur sa trajectoire. " (p. 91). Le défi lancé à l'intervenant, c'est de saisir avec justesse le degré d'intensité des émotions du jeune qui se confie, la tonalité de son appel, les traits caractéristiques de son état de conscience, les particularités de sa perception d'autrui et de luimême, la nature de sa souffrance et la spécificité de son rapport à la mort (voir à cet effet les " tableaux récapitulatifs "à la fin du volume : p. 229-238). Si ce travail d'empathie est accompli avec le souci de la précision, l'intervenant peut savoir à quelle enseigne loge l'adolescent et, par conséquent, dire le mot juste et poser le geste adéquat. " Chaque phase constitue un moment particulier, un espace de vie, un univers en soi. [...] La prévention aura donc deux visages : limiter immédiatement la souffrance vécue et ménager dans la phase où l'adolescent se trouve un échange qui ouvre une dynamique de soins. " (p. 111).

\section{Le " psymal "}

\section{et la "pensée focale "}

Aux yeux des auteurs, Grand Bleu, film culte de Luc Besson est emblématique de la distance qui existe entre la culture des adolescents, qui y ont détecté un reflet de leur souffrance, et les adultes, qui ont éprouvé un malaise devant un film " sans scénario, sans intrigue, ni suspense ". On s'en doute, le choix du titre du présent volume est fortement inspiré par le titre du film. L'adolescence est marquée par une métamorphose physique et psychique. " L'adolescent est un être en mouvement et c'est ce mouvement qui le définit. [...] Ce n'est pas l'adolescent qu'il faut observer, mais sa trajectoire. "( $p$ 145). Cette métamorphose est faite d'émerveillement et de nouveauté, mais aussi de souffrance. Dans ce contexte, les auteurs reprennent la notion de "psymal ", développée par l'américain Edwin Schneidman dans Le tempérament suicidaire : "Le psymal se rapporte à la douleur, l'angoisse, le regret, la souffrance, la misère qui nous torturent l'esprit. C'est la souffrance intensément ressentie de la honte, de la culpabilité, de l'humiliation, de la solitude, de la perte, de la tristesse, de la terreur de vieillir ou de mal mourir, etc. Quand elle est ressentie, sa réalité introspective est indéniable. " (p. 160). Les auteurs élaborent aussi la notion de " pensée focale " qui fait qu'un individu concentre son intérêt sur un point unique de la réalité. Cette fixation change sa perception du reste du monde, comme elle le change aussi et lui fait oublier sa douleur. Chez l'adolescent suicidaire, l'objet de cette focalisation est la mort. " La trajectoire suicidaire est donc marquée par l'installation progressive d'une anesthésie psychique et physique facilitant le passage à l'acte. Les solutions préventives doivent passer par la mise en place d'un réveil, d'une sortie de la transe. " (p. 170).

\section{Que faire?}

Les auteurs proposent des stratégies sommaires d'intervention auprès des adolescents pour chacune des phases de l'itinéraire vers la mort. C'est sans doute la partie du présent volume qui risque de décevoir le plus les lecteurs. Une richesse inouie de connaissances sur le profil psychologique de l'adolescent hanté par l'idée de la mort aboutit à quelques conseils pratiques sans doute pertinents, mais si pauvres par rapport à la grande construction de l'esprit qui domine l'ensemble de ces pages. Ceci s'explique pourtant, car il y a une distance énorme, d'une part, entre l'idéal d'une écoute empathique et d'une action correspondante et, d'autre part, les incertitudes de nos observations et de nos interventions. En plus, cette construction logique, fondée sans doute dans la réalité observée et donc valable, fait penser aux stades du développement moral selon Lawrence Kohlberg ou aux étapes du deuil selon Élisabeth Kübler-Ross. Elle est révélatrice d'une évolution qui s'effectue par étapes et elle indique les balises d'une trajectoire. Elle suggère une voie sans autre prétention que celle d'une interprétation parmi d'autres de la mouvance de l'âme humaine à travers les aléas de l'existence, avec des avances et des reculs. Cette séquence, observée et présentée selon un ordre chronologique, ne doit donc pas être prise trop à la lettre, car elle risque d'enfermer l'adolescent en crise et l'intervention de l'adulte écoutant dans des cases trop étroites, plus ou moins validées et vérifiables. Cette réserve, exprimée avec la pudeur de celui qui ne sait pas trop, n'est qu'une interrogation critique que nous soumettons aux auteurs dont nous avons pu apprécier grandement l'approche empathique. Celle-ci naît d'une curiosité bienveillante ou d'un souci fondamental, celui de savoir ce qui se passe exactement dans l'esprit et le cœur de la jeune fille et du garçon aux prises avec l'idée ou le désir de la mort. Une toute dernière question sans doute déplacée : en fermant ce livre, on n'échappe pas à une certaine impression de dolorisme. Sans vouloir s'adonner à quelque minimalisme indécent, on peut se demander si la souffrance des adolescents est si généralisée. C'est à eux de nous le dire.

Éric Volant
André Compte-Sponville

Présentations de la philosophie

Paris, Albin Michel, 2000,

219 pages.

Andri Comte-Sponville Presentalions de la philosophice

albin Michel

Ce livre est la reprise de douze carnets indépendants et originellement publiés dans une collection d'initiation à la philosophie. Ces carnets, revus et sensiblement augmentès, constituent maintenant les douze chapitres du présent ouvrage. La modestie du propos reste toujours la même: il s'agit de douze portes d'entrée dans la philosophie. L'auteur a donc renoncé aux notes en bas de pages et a préféré commenter une brève bibliographie (p. 207-217). Ce choix est judicieux, car ces notes auraient alourdi considérablement l'ensemble de ses réflexions. En effet, plusieurs philosophes sont mentionnés à chaque page ou presque. Parmi les noms qui reviennent le plus souvent, on peut citer Kant (bien que Compte-Sponville affirme, à la page 139, ne pas être kantien), Spinoza, Montaigne et Alain, pour les Modernes, puis Platon et Aristote, pour les Anciens. En ce qui concerne les philosophes orientaux, seul Svâmi Prajnânpad, sage de I'Inde auquel Compte-Sponville a déjà consacré un livre, est cité à deux reprises (p. 183-184). Cette brève enquête sur les auteurs signalés par Compte-Sponville indique bien que la philosophie visée par le titre du livre est celle de l'Occident, ce qui ne présuppose aucunement, de l'aveu même de l'auteur, que la philosophie soit exclusivement grecque et occidentale (p. 15).

Les douze thèmes retenus sont les suivants: la morale, la politique, I'amour, la mort, la connaissance, la liberté, Dieu, l'athéisme, l'art, le 
temps, l'homme et la sagesse. Seul le thème de la mort (p. 59-68), ce génie inspirateur de la philosophie, retiendra ici mon attention.

Bien que l'amour soit pour Compte-Sponville le seul problème philosophique vraiment sérieux ( $p$. 48), philosopher n'en reste pas moins, selon l'expression qu'il emprunte à Montaigne, apprendre à mourir. Mais ce n'est apprendre à mourir que parce que c'est apprendre à vivre, et parce que la mort fait partie de la vie. Ceci dit, comme toute pensée sur la mort, celle de Compte-Sponville instruit moins sur la mort que sur ses propres présupposés philosophiques à l'égard de l'existence humaine. En effet, tout en sachant qu'il simplifie à l'extrême, CompteSponville répartit les réponses face à la mort en deux camps: les uns qui disent que la mort n'est rien (un néant, strictement); les autres qui affirment qu'elle est une vie, ou la même continuité, purifiée, libérée. Pour l'auteur, c'est deux façons de nier la mort: comme néant, puisque le néant n'est rien et comme vie, puisque la mort, alors, en serait une. Par ailleurs, précise-til, seuls les athées et les philosophes matérialistes, donc ceux qui appartiennent au premier camp, prennent la mort au sérieux (p. 61). Cette dernière affirmation n'est pas étonnante de la part d'un penseur qui affirme ne pas croire en Dieu (p. 97-111) et qui se déclare athée ( $p$. 113-129), mais elle n'en reste pas moins discutable, d'autant plus qu'elle s'appuie sur une simplification abusive des deux seules réponses possibles qu'il entrevoit face à la mort. Or, l'histoire des religions permet difficilement de cataloguer tous les êtres humains dans l'un ou l'autre de ces camps. Par exemple, un philosophe juif comme Qohélet - pour ne nommer que celui-ci - n'appartient véritablement à aucune de ces deux catégories. De son côté, la psychanalyse freudienne ne saurait davantage endosser ce jugement de Compte-Sponville, puisqu'elle est convaincue que personne ne croit à sa propre mort ou, ce qui revient au même, que chacun, dans son inconscient, est persuadé de sa propre immortalité. Dit autrement, selon la perspective freudienne, il est difficile d'affirmer qu'un croyant prend forcément la mort moins au sérieux qu'un athée. À ce sujet, une comparaison de la philosophie marxiste, qui conçoit la mort comme une dure victoire de l'espèce sur l'individu, avec la méditation bouddhiste sur le cadavre en état de décomposition pourrait peut- être même laisser croire le contraire...

Ces quelques remarques, qu'il m'est impossible de développer à l'occasion d'un bref compte rendu, illustrent simplement que la mort désigne moins un contenu philosophique que la fonction qui fait de la philosophie une interrogation toujours ouverte. Bien entendu, Compte-Sponville n'entend pas dire le dernier mot sur la mort, ni sur aucun des douze thèmes abordés. Au contraire, tout en affichant ses propres convictions, il vise plutôt à ce que son lecteur apprenne à philosopher, dans le but, non pas de faire des livres, mais de vivre de façon plus lucide, plus libre, plus heureuse et plus sage. Or, s'il est vrai que c'est dans les livres de philosophie qu'on apprend à philosopher (p. 179), celui de CompteSponville est sans contredit un de ces ouvrages où le vaste public, auquel il est d'abord destiné, pourra faire un tel apprentissage.

Jean-Jacques Lavoie

\section{BIBLIOGRAPHIE}

Bernard Jobin

\section{LIVRES ET ARTICLES}

ARFEUX-VAUCHER, Geneviève, La vieillesse et la mort dans la littérature enfantine de 1880 à nos jours, Paris, Éditions Imago, 1994.

ALTSCHUL, S., Childhood Bereavement and Its Aftermath, Madison, Conn., International Universities Press, 1988

AMAR, Nadine, "Le deuil ", dans Monographies de la Revue française de psychanalyse, sous la direction de Nadine AMAR, Catherine COUVREUR, Michel HANUS, Paris, Presses universitaires de France, 1994.

ANTHONY, Sylvia, The Discovery of Death in Childhood and After, London, A. Lane, 1971, 280 pages.

ASPINALL, S. Y., " Educating Children to Cope with Death : A Preventive Model ", Psychology in the schools, vol. 33, no 4, 1996, p. 341-349.

BACQUÉ, M.-F., Deuil et santé, Paris, Odile Jacob, 1997.

BACQUÉ, Marie Frédérique, Mourir aujourd'hui, Les nouveaux rites funéraires, Paris, Odile Jacob, 1997.

BACQUÉ, M.-F., HANUS, M., Le deuil, Paris, PUF, coll. "Que saisje ? ", 2000.

BAYLE, G., Le deuil, Paris, PUF, 1995, 174 pages.
BEARISON, J. D., They Never Want to Tell You, Cambridge, Mass., Harvard University Press, 1991.

BIRENBAUM, L. K., " Assessing Children's and Teenagers' Bereavement when a Sibling Dies from Cancer : A Secondary Analysis ", Child Care Health and Development, vol. 26, no 5, 2000 , p. 381-400.

BOURGEOIS, Marc Louis, Le Deuil : clinique et pathologie, Paris, Presses universitaires de France, 1996.

BOURRAT, M. M., " L'enfant confronté au décès fraternel ", Neuropsychiatrie de l'enfance et de I'adolescence, vol. 47, no 1-2, 1999 , p. 100-109.

BOWLBY, J., Attachment and Loss. Loss, Sadness and Depression, London, Hogarth Press, 1991.

BOYD, N., "Beyond the Innocence of Childhood, vol. 1, Factors Influencing Children and Adolescents' Perceptions and Attitudes Toward Death, vol. 2, Helping Children and Adolescents Cope with Life-Threatening Illness and Dying, vol. 3, Helping Children and Adolescents Cope with Death and Bereavement ", Omega Journal of Death and Dying, vol. 40, no 2, 1999, p. 395-398.

BROUSSOLLE, P., "Bereavements in Our Lifetime. Loss and Separation in Adults and Children ", Évolution psychiatrique, vol. 65, no 2, 2000, p. $445-446$.

BUREAU, Serge, LACHAINE Richard, Aujourd'hui la mort, sous la direction de Serge Bureau, Québec, Fides, 1996

CAROLI, F., GUEDJ, M.-J., Le suicide, Paris, Flammarion, coll. "Dominos», 1999.

CASTELLAN, Y., Les grands-parents ces inconnus, Paris, Bayard Éditions, coll. "Psychologie ", 1998.

CASTRO, Dana, La mort pour de faux et la mort pour de vrai, Paris, Albin Michel, 2000, 203 pages.

CEREL, J., FRISTAD, M.A., WELLER, E., WELLER, R.A., "SuicideBereaved Children and Adolescents : A Controlled Longitudinal Examination", Journal of the American Academy of Child and Adolescent Psychiatry, no 38,1999, p. 672-679.

CHANTELOIS, Mariane, Perception et compréhension du concept de la mort, dans un livre illustré, par la conduite du récit chez l'enfant, Rapport de stage de maîtrise en éducation, Université du Québec à Montréal, 1989, 115 pages.
Child and Adolescent Psychiatry A Comprehensive Textbook, edited by M. LEWIS, Baltimore, Williams \& Wilkins, 1991, 1282 pages.

Child and Adolescent Psychiatry Continuing Education Review : 639 Essay Questions and Referenced Answers, edited by G. PIROOZ SHOLEVAR, Flushing, N.Y., Medical Examination Pub. Co., 1977.

Childhood and Death, edited by H. WASS and C. A. CORR, Washington, Hemisphere Pub. Corpages., 1984, 392 pages.

Clinical Child Psychiatry, edited by W. M. KLYKYLO, J. KAY, D. RUBE, 1st ed., Philadelphia, W. B. Saunders Co., 1998.

COLLECTIF, Parents orphelins témoignages de parents à la suite du décès de leur enfant, Montréal, L. Courteau, 1990.

COSLIN, G.-P., Les adolescents devant les déviances, Paris, PUF, 1996.

DELAROCHE, P., Doit-on céder aux adolescents ?, Paris, Albin Michel, 1999.

DELAROCHE, P., Adolescents à problèmes, Paris, Albin Michel, 2000.

DELBES, A., "Perception de la fin de vie par les enfants: La mort ", Gérontologie et société, no 90, 1999 , p. 27-33.

DIATKINE, G., " Deuil et inhibition intellectuelle chez le jeune enfant ", Bulletin de Psychologie, vol. 38 , no $370,1984-1985$, p. 491494.

DEBRA, A., " La mort et le deuil à l'école ", dans Études sur la mort, Paris, L'esprit du temps, 1999.

DOLTO, F., Parler de la mort, Mercure de France, Gallimard, 1998.

DONDERS, J., “ Bereavement and Mourning in Pediatric Rehabilitation Settings ", Death Studies, vol. 17, no 6, 1993, p. 517-527.

DOUESNARD, Suzanne, "Est-ce que tu savais que je vais mourir?", Frontières, La mort chez l'enfant et l'adolescent, vol. 2, no 1, printemps 1989, p. 30-35.

DOWDNEY, L., " Annotation Childhood Bereavement Following Parental Death ", Journal of Child Psychology and Psychiatry and Allied Disciplines, vol. 41, no 7 , p. $819-830$.

DYREGOV, A., Grief in Children, London, Jessica Kingsley Publishers, 1990.

ENCREVÉ-LAMBERT, M.-H., La mort, Paris, Bayard Éditions, 1999. 